INPLASY

PROTOCOL

To cite: Sun et al. Functional

Changes in the Brain of

bulimia nervosa: a protocol for

systematic review and meta

analysis. Inplasy protocol

202130024. doi:

10.37766/inplasy2021.3.0024

Received: 07 March 2021

Published: 08 March 2021

Corresponding author:

Yi-ming Sun

sunyiming93@foxmail.com

Author Affiliation:

Chengdu Eighth People's

Hospital (Geriatric Hospital of

Chengdu Medical College)

Support: None.

Review Stage at time of this submission: Preliminary

searches.

Conflicts of interest:

None declared.

\section{Functional Changes in the Brain of bulimia nervosa: a protocol for systematic review and meta analysis}

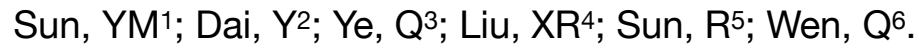

Review question / Objective: To summarize and meta-analyze studies on changes in fMRI, ALFF, and ReHo in patients with bulimia nervosa.

Condition being studied: Specifically, BN is impulsive / compulsive eating disorder with altered reward sensitivity and food-related attentional biases. Compared to HCs, BN patients have impaired response inhibition and general impairments in inhibitory control, when faced with eating disorder-related stimuli. Evidence suggests that function changes in the prefrontal, insular cortex, orbitofrontal cortex (OFC) and striatum of BN patients. and the severity of symptoms in BN patients is related to the diminished frontostriatal brain activation.

INPLASY registration number: This protocol was registered with the International Platform of Registered Systematic Review and Meta-Analysis Protocols (INPLASY) on 08 March 2021 and was last updated on 25 March 2021 (registration number INPLASY202130024).

\section{INTRODUCTION}

Review question / Objective: To summarize and meta-analyze studies on changes in fMRI, ALFF, and ReHo in patients with bulimia nervosa.
Rationale: Recurrent binge eating is a core diagnostic criterion for bulimia nervosa (BN), and also is a common feature in anorexia nervosa-binge purge type (AN-BP) and other specified feeding and eating disorder (OSFED). BN is noted social and health consequences that typically arise in 
later adolescent and young adult years, Over one in five individuals with BN will attempt suicide during their life, with factors relating to Psychiatric comorbidity, particularly with depression, anxiety disorders.

Condition being studied: Specifically, BN is impulsive / compulsive eating disorder with altered reward sensitivity and food-related attentional biases. Compared to HCs, BN patients have impaired response inhibition and general impairments in inhibitory control, when faced with eating disorderrelated stimuli. Evidence suggests that function changes in the prefrontal, insular cortex, orbitofrontal cortex (OFC) and striatum of BN patients. and the severity of symptoms in BN patients is related to the diminished frontostriatal brain activation.

\section{METHODS}

Search strategy: A preliminary systematic search was performed using Cochrane Library, PubMed, Embase, and Web of Science, CNKI from inception to January 3, 2021. Two researchers will independently conduct the search, screen titles and abstracts for study selection and extract the relevant data. A third senior researcher will review articles should disputes arise over study inclusion.

Participant or population: Adolescents and adult patients diagnosed with $B N$ according to the DSM-5 or any recognized diagnostic criteria.

\section{Intervention: None.}

Comparator: Datasets included within the analysis will include a comparison of Bulimia nervosa patients to healthy adults.

Study designs to be included: Observational studies or baseline information of RCTs.

Eligibility criteria: 1 . Studies of comparing functional cerebral alterations of bulimia nervosa with that of healthy controls will be included. 2. Adolescents and adult patients diagnosed with BN according to the DSM-5 or any recognized diagnostic criteria. 3. Whole-brain results in three-dimensional coordinates $(x, y, z)$ of changes in standard stereotactic space (Talairach or MNI) were reported. 4. Thresholds for significance corrected for multiple comparisons or uncorrected with spatial extent thresholds were used. 5. The study is available in the English language. Criteria for exclusion. 1. Studies only reporting region of interests (ROIs) findings were excluded. 2. Studies using coordinates relative to analyze employing small volume corrections (SVC) in preselected ROls were excluded.

Information sources: We will search electronic databases including the Cochrane Library, Web of Science, PubMed, EMBASE, China National Knowledge Infrastructure (CNKI).

Main outcome(s): Separate functional brain response abnormalities will be conducted with the signed differential mapping (SDM). This method bases on using the peak coordinates to recreate, for each study, a map of the effect sizes of the differences between patients and controls, and then conducting a standard random-effects variance-weighted meta-analysis in each voxel.

Additional outcome(s): None.

Data management: The references obtained during the searches will be screened for relevance, and those identified as being potentially eligible will be fully assessed against the inclusion/ exclusion criteria. Two reviewers (DY and YQ) will independently double check the eligibility of the included studies and extract data by entering details into a predefined data acquisition form. This acquisition form will include four main domains: citation information (title, author list, source of publication, year of publication, first author's name and affiliation, country, sponsor), design (design, participants, trial methods, duration, intervention details, caregiver information), results (outcome measures, adverse events) and conclusion. Any 
discrepancy noticed in the process of data cross-checking will be resolved through discussion and the suggestion of a third reviewer (SRR).

Quality assessment / Risk of bias analysis: The risk of bias for each included trial will be evaluated using NOS (NewcastleOttawa Scale) for assessing risk of bias in randomised trials.

Strategy of data synthesis: Seed-based d Mapping (SDM) software will be used for the Effect Size-SDM (ES-SDM) analysis. Peaks coordinates of activation will be extracted from the literature and checked by both authors to reduce risk of error. Peak coordinates not in (Montreal Neurological Institute) MNI space will be converted using coordinate mapping software. Standard processing steps will be used in accordance with software documentation.

Subgroup analysis: We plan to explore reasons for heterogeneity between studies, and check the results through sensitivity analyses. If sufficient trials are included, we will explore the following potential sources of heterogeneity using subgroup analyses or meta-regression: 1 . Studies with low risk of bias compared to trials with high risk of bias; 2. Methods: alffreho; 3. Scan T:1.53.0; 4. According to the results of this review.

Sensitivity analysis: We will carry out the sensitivity analysis to guarantee the stability and reliability of the research results.

Language: English.

Country(ies) involved: China.

Other relevant information: None.

Keywords: bulimia nervosa; fMRI.

Dissemination plans: This review does not require ethical approval duo to data that we will not endanger the individual's privacy or compromise their rights.
Contributions of each author:

Author 1 - Yi-ming Sun.

Author 2 - Yu Dai.

Author 3 - Qing Ye.

Author 4 - Xin-ru Liu.

Author 5 - Ran Sun.

Author 6 - Quan Wen. 